\title{
FEMUR METASTASIS SECONDARY TO A CARCINOMA OF THE UTERINE CERVIX (CASE STUDY)
}

\author{
doi: 10.2478/rojost-2018-0031 \\ D. Popescu ${ }^{1}$, C. Panaitescu ${ }^{1}$, R. Nedelcu ${ }^{1}$, Șt. Trifu ${ }^{1}$, C. Cîrstoiv ${ }^{1,2}$ \\ ${ }^{1}$ Orthopedics and Traumatology Department, University Emergency Hospital, Bucharest, Romania \\ 2"Carol Davila" University of Medicine and Pharmacy, Bucharest, Romania
}

Purpose. The purpose of this study is to correlate the existence of a unique bone metastasis of the femur, secondary to a carcinoma of the uterine cervix, the treatment plan, and the recovery of the patient.

Materials and method. From previous studies, it is known that the prevalence of unique bone metastasis of the femur secondary to carcinoma of the uterine cervix is very low, in most cases the patients having multiple metastases or in other organs. The case presents a 40 -year-old patient who had surgery 2 years before for a carcinoma of the uterine cervix. After the surgical treatment, the patient received chemotherapy and radiotherapy. Nowadays, the patient exhibits pain in the left hip. Following the clinical and paraclinical protocols, an incisional biopsy was made. The histopathologic result was metastasis from the uterine cervix squamous cell carcinoma. The surgical treatment consists of segmentary resection and total hip arthroplasty with tumor reconstruction prosthesis.

Results. After total hip arthroplasty with tumor reconstruction prosthesis, we were able to make a segmentary resection in oncological limits, with the complete excision of the metastasis, the patient being able to start the recovery the next day after the surgery.

Conclusions. As a surgical indication, the tumor prosthesis represents the best option in the segmentary resections of tumors, this allowing a reconstruction and an early recovery.

Keywords: metastasis, squamous cell carcinoma, tumor prosthesis 